\title{
Lattice gauge theory and physics beyond the standard model
}

\author{
Joel Giedt ${ }^{* \dagger}$ \\ Department of Physics, Applied Physics and Astronomy, Rensselaer Polytechnic Institute \\ 110 8th Street, Troy, NY 12180 USA \\ E-mail: giedtjerpi.edu
}

Over the last several years, lattice field theory methods have been applied to numerous theories beyond the standard model. While steady progress has been made in the area of lattice supersymmetry, most of the recent work has concentrated on lattice studies of a composite Higgs mechanism, where fermion condensation due to a new strong interaction spontaneously breaks electroweak symmetry. We describe some of the successes and controversies in this field in the last few years. The need for larger scale studies involving appreciable lattice volumes is highlighted. We also comment briefly on the continued relevance of these studies of a new strong interaction in light of the recent experimental discovery of a Higgs boson at $125 \mathrm{GeV}$. It is argued that there still remains the possibility that this particle is not an elementary scalar, but may be a composite state of a new strong interaction. Indeed, it is incumbent upon high energy physicists to fully explore this scenario before ruling it out. The most important task of beyond the standard model lattice gauge theory over the next few years will be to answer the question: Is it possible to have a scalar state, such as a dilaton $\sigma$, that is much lighter than the rest of the "hadronic" spectrum of this new strong interaction, which should be near a TeV? We mention some early work in this direction, both on and off the lattice. It is interesting that theories of a warped extra dimension (the Randall-Sundrum scenarios) play an important role in this regard, and that states in the holographic dual may be a mixture of composite and elementary degrees of freedom. In particular, we point to the implications of the observed production $g g \rightarrow \sigma$ and decays $\sigma \rightarrow \gamma \gamma, Z Z$, for TeV scale physics beyond the standard model.

The 30 International Symposium on Lattice Field Theory - Lattice 2012,

June 24-29, 2012

Cairns, Australia

\footnotetext{
${ }^{*}$ Speaker.

$\dagger$ This work was supported in part by the Dept. of Energy, Office of Science, Office of High Energy Physics, Grant No. DE-FG02-08ER41575.
} 


\section{Introduction}

Dynamical electroweak symmetry breaking through a new strong interaction has been the focus of a flurry of work over the last few years. This will be the topic with which I will be mainly concerned in this review. Unfortunately it is not possible to also cover recent advances in many other applications of lattice gauge theory to physics beyond the standard model. Other examples include supersymmetry, matrix models, quantum gravity, extra dimensions, as well as many efforts to obtain accurate predictions of the standard model in order to look for deviations. To this list could be added a large number of works that are not devoted to quantum chromodynamics per se, but that study quantum field theory or many body theory using lattice field theory techniques.

The theory of the elementary standard model Higgs boson has two problems associated with it: the gauge hierarchy problem and the triviality problem. In the first case, radiative corrections to the Higgs mass-squared $m^{2}$ are quadratically divergent, and so the natural scale for $m$ is the one where new physics takes over, the cutoff scale $\Lambda_{\text {cutoff }}$. This could be the grand unified scale $\left(\gtrsim 10^{15} \mathrm{GeV}\right.$ ), or it could be the Planck scale $10^{19} \mathrm{GeV}$. Whatever the exact number, it is quite a bit higher than the electroweak scale $100 \mathrm{GeV}$, which poses a significant mystery: why is $m$ of order $100 \mathrm{GeV}$ ? There are many possible resolutions to this problem, including electroweak scale supersymmetry, technicolor and warped extra dimensions. In each case, the hierarchy between the cutoff scale and the electroweak scale is generated in a natural way that eliminates fine-tuning. In the cases of supersymmetry and technicolor, this is achieved dynamically through dimensional transmutation: the theory is weakly coupled at a high scale, but due to running of the gauge coupling a dynamical scale is generated at low energies, which then becomes an order parameter that sets the scale of electroweak physics. In supersymmetry this is achieved through new strong dynamics in a hidden sector and some mechanism of mediation to the observable sector. In addition, the electroweak scale is rendered technically natural because quadratic corrections are cancelled at high energies, where soft supersymmetry breaking can be neglected. In technicolor this is achieved through new strong dynamics with techniquarks, fermions that are charged under the electroweak gauge group $S U(2)_{L} \times U(1)_{Y}$. In both cases, the fact that strongly coupled gauge theories form condensates that can break symmetries spontaneously is crucial to the scenarios considered. The warped extra dimension (Randall-Sundrum) scenario at first look seems quite orthogonal: the hierarchy is determined by a geometric factor. However, this set-up also has a holographic interpretation in terms of a four-dimensional theory with a new strong interaction. Thus even in this case, the physics of nonperturbative, dynamically generated scales is something we would like to understand more fully.

Triviality also comes with an interesting story, and ought to be addressed by some means if one is to have a complete theory. Here the problem is that one cannot remove the cutoff, since if one tries, all couplings in the infrared will go to zero (a trivial theory). In the case of supersymmetric models, the quartic self-interaction of scalars such as the Higgs fields becomes a function of gauge couplings, so the triviality problem is replaced by the lack of asymptotic freedom in the 
$S U(2)_{L} \times U(1)_{Y}$ gauge theory. (In supersymmetric extensions to the standard model, $S U(2)_{L}$ is no longer asymptotically free, due to the numerous new particles that are charged under this group.) This can in principle be addressed by embedding the standard model gauge group into a grand unified group such as $S U(5), S O(10)$ or $E_{6}$, provided the representations in the theory are such that it is asymptotically free. However, in order to obtain realistic physics at low energies, especially if Yukawa coupling unification is also assumed, a large number of representations must be added (an unwieldy Higgs sector). In that case, the grand unified theory is not asymptotically free and so there is again a triviality problem. This is resolved if one proceeds to the next level of unification, combining the gauge theory with gravity in the context of string/M theory. Since superstring theory is finite, the cutoff is no longer required and the triviality problem is solved. Of course the price one pays is that string/ $\mathrm{M}$ theory has a vacuum selection problem: there is a vast number of different string vacua (differentiated by gauge groups, representations and even the number of large dimensions) and no dynamical mechanism to select our universe as the correct one. This has even led some physicists to speculate on anthropic solutions to the this problem [1]. See however counterarguments in the recent review [2].

In the case of warped extra dimensions, the existence of a warped geometry with an ultraviolet brane and an infrared brane strongly suggests Type IIB superstring theory, with stacks of D3 branes providing the warped geometry in the near-horizon limit. The flavors in the bulk can be interpreted in terms of D7 branes, and the ultraviolet brane can be an effective description of a six-dimensional Calabi-Yau manifold at the large radius end of the throat [3]. Hence the triviality problem is again solved by a unified theory of gravity and gauge forces.

Technicolor models are more modest, though there is no reason why they cannot be embedded into string/M theory, and through the holographic principle may even be dual to the warped extra dimension scenario. To address fermion masses and flavor physics, the technicolor theory is embedded into an extended technicolor model. Generally it is assumed that this theory is asymptotically free (however, in the example of [4] there is a U(1) factor, which again will have a triviality problem). Since the Higgs is no longer elementary, and the underlying theory is an asymptotically free gauge theory, the triviality problem is solved.

The focus of this review is models that break electroweak symmetry $S U(2)_{L} \times U(1)_{Y} \rightarrow$ $U(1)_{E M}$ through a composite Higgs mechanism, such as fermion condensation in technicolor models. Since these are supposed to be theories with a phenomenological application, it is important to address the current experimental situation, and to contrast it with what is the case for the other models that have been mentioned. There is an apparent discovery of a Higgs boson by ATLAS [5] and CMS [6], experiments at the Large Hadron Collider at CERN. This comes from $5 \sigma$ excesses observed near $125 \mathrm{GeV}$ in the combined $2 \gamma$ and $\mathrm{ZZ} \rightarrow 4 \ell$ channels. The rates are consistent with a standard model Higgs, given the present uncertainties. Other channels have too large of backgrounds to add much to the story for the integrated luminosity that has been analyzed so far. If indeed the Higgs boson has been discovered, the experimental significance should increase with the 2012 running that will be completed at the beginning of 2013. More data and analysis is needed 
in order to be more definitive in more channels; the detailed properties of this new particle must be explored in order to conclude that it is indeed the standard model Higgs boson. This raises the question of whether or not it is possible for theories with a composite Higgs mechanism to yield a state with experimental behavior that is similar to an elementary standard model Higgs boson with a mass of $125 \mathrm{GeV}$. Because the compositeness scale is of order $4 \pi f$, and $f \approx v=246 \mathrm{GeV}$, it is necessary to explain a hierarchy of scales in a dynamical fashion. The way that this would occur is through the "technidilaton," a pseudo-Nambu-Goldstone boson associated with spontaneous breaking of scale invariance in a nearly conformal gauge theory. If the $\beta$ function is quite small when the coupling reaches the value at which fermion condensation sets in, then scale invariance is an approximate symmetry that is spontaneously broken. Thus in some models of "walking technicolor," it may be possible to have a light scalar. This scenario has recently attracted a lot of attention, with various opinions about its viability. See for example the effective field theory approach and holographic dual analysis of [7]. It is amusing that supersymmetry could actually rescue technicolor in this approach! Obviously this assertion needs to be proven by a first principles approach, since naive dimensional analysis in a nearly conformal gauge theory may not be valid, and lattice gauge theory is an ideal tool for doing this. However, it is quite a demanding task to study a nearly conformal theory on the lattice due to the large hierarchy of scales $a \ll 1 / f \ll L$ (where $a$ is the lattice spacing and $L$ is the extent of the lattice in any of the four spacetime directions), and in fact it is difficult to be sure whether or not one even has a candidate such theory (as will be seen in much of this review below). There is, however, no shortage of claims by phenomenologists for the existence of technidilatons, as well as critics of this proposal who say it is impossible or even inconsistent. One interesting thing to note is that if the holographic principle is valid, then the technidilaton is dual to the radion of warped extra dimensional theories. In this case one can study the prospects for a light dilaton in a weakly coupled five-dimensional dual theory. This has been done, and it has been found that significant fine-tuning is required unless the theory is supersymmetric [7].

\section{Theory background}

\subsection{Walking behavior versus infrared fixed point}

The evolution of the running gauge coupling $g=g(\mu)$ is given by the $\beta$ function, which in perturbation theory is a power series

$$
\frac{d g}{d \ln \mu}=\beta(g)=-b_{1} g^{3}+b_{2} g^{5}+\mathscr{O}\left(g^{7}\right)
$$

In the case where $b_{1}, b_{2}>0$, we find a nontrivial fixed point at two loops, $\beta\left(g_{*}\right)=0, g_{*} \neq 0$, where the $\mathscr{O}\left(g^{7}\right)$ term is neglected. This phenomenon was noticed early in the study of nonabelian gauge theories [8], and explored more fully in the well-known work of Banks and Zaks [9]. Provided $b_{1}>0$, the theory is asymptotically free and so starting from the high scale the theory evolves down starting at $g \ll 1$ to the larger value $g_{*}$ where the infrared fixed point is reached. In the canonical example of SU(3) gauge theory with $N_{f}$ massless fermions in the fundamental representation, this 
fixed point behavior occurs for $9 \leq N_{f} \leq 16$, which is the two loop conformal window. This is not exactly true because the coupling may first reach a critical value $g_{c}$ at which fermion condensation occurs, modes decouple, and the theory confines instead of approaching the fixed point. Such an avoidance of the fixed point occurs if $g_{*}>g_{c}$. Often this is spoken of in terms of a dynamical mass being generated for the fermions when condensation occurs. One can of course go to higher loop order and ask if this behavior persists. Ultimately one exchanges perturbation theory for a nonperturbative tool, such as the lattice, and in that case one determines whether or not the infrared fixed point is a part of the full theory. In supersymmetric quantum chromodynamics, this question can be answered exactly and reliably using continuum methods, due to fact that the exact $\beta$ function is known in a particular scheme - the NSVZ result, and using arguments due to Seiberg. The range of parameters, especially the number of fermion flavors $N_{f}$, over which this infrared fixed point phenomenon occurs is called the conformal window.

Proximity to the conformal window is important to realizing the scenario of walking dynamics, used in so-called walking technicolor. In this class of models, the fermion masses in the standard model are given by exchange of the extended technicolor (ETC) gauge boson with the condensate of technifermions at the ETC scale:

$$
m \sim \frac{\langle\bar{\psi} \psi\rangle_{\mathrm{ETC}}}{M_{\mathrm{ETC}}^{2}}
$$

If there is a walking coupling between the scale $M_{\mathrm{ETC}}$ and the technicolor condensation scale $\Lambda_{\mathrm{TC}} \sim\left|\langle\bar{\psi} \psi\rangle_{\mathrm{TC}}\right|^{1 / 3}$, then the condensate at the two scales will be related by renormalization group evolution,

$$
\langle\bar{\psi} \psi\rangle_{\mathrm{ETC}} \approx\left(\frac{M_{\mathrm{ETC}}}{\Lambda_{\mathrm{TC}}}\right)^{\gamma}\langle\bar{\psi} \psi\rangle_{\mathrm{TC}}
$$

where $\gamma$ is the anomalous mass dimension at the approximate fixed point $g_{*}$, a nontrivial local minimum of $|\beta(g)|$ where $\left|\beta\left(g_{*}\right)\right| \ll 1$. The quantity $\langle\bar{\psi} \psi\rangle_{\mathrm{TC}} \sim \Lambda_{\mathrm{TC}}^{3}$ is fixed by $m_{W} \sim \Lambda_{\mathrm{TC}}$. On the other hand, the fermion masses are determined by $\langle\bar{\psi} \psi\rangle_{\mathrm{ETC}}$. Since $M_{\mathrm{ETC}} \gg \Lambda_{\mathrm{TC}} \sim m_{W}$ in order to suppress flavor-changing neutral currents, having $\gamma \approx 1$ enhances the fermion masses as needed.

In order to construct such models, it is necessary to find walking dynamics in "theory space," and this has been the focus of much recent lattice work. To do this it is necessary to distiguish between behavior in the presence of an infrared fixed point (conformal dynamics) versus behavior with a slowly running coupling but spontaneous chiral symmetry breaking and confinement at large distance scales. We will not describe the theory behind fitting to spontaneous chiral symmetry breaking, since it is quite standard from quantum chromodynamics. Another rather conventional approach is to identify a finite temperature transition at which the theory deconfines and chiral symmetry is restored. Below the critical temperature there is a chiral condensate; hence the theory cannot be conformal. A few studies have been performed in this direction. However, the fits to conformal theory may be less familiar, so we summarize these next. 


\subsection{Conformal fits}

In order to assess the likelihood that a theory flows into an infrared fixed point, it is common practice to attempt to fit simulation results to the conformal hypothesis. Since virtually all spectral studies are performed at a nonzero fermion mass $m$, we are particularly interested in mass deformed conformal field theory, which has been addressed in Refs. [10-13]. We begin with infinite volume results. There, masses and decay constants have the hyperscaling behavior

$$
M, F \sim m^{1 /(1+\gamma)}
$$

with a universal exponent $\gamma$ in all "channels." From [12] we also have for the chiral condensate,

$$
\langle\bar{\psi} \psi\rangle \sim m^{(3-\gamma) /(1+\gamma)}
$$

Another interesting quantity that could be measured is the gluon condensate

$$
\left\langle\operatorname{Tr} F_{\mu \nu} F_{\mu v}\right\rangle \sim m^{4 /(1+\gamma)}
$$

However, this operator mixes with the identity operator so there is a large divergent contribution that must be subtracted off before the fit can be performed. There are some thoughts that using Wilson flow (a.k.a. gradient flow) would allow for a better determination of this quantity, though the details behind this still need to be worked out.

Lattice simulations are performed in finite volume, so one can ask: What are the finite volume versions of these formulae? One answer to this question is provided by the theory of finite volume corrections where for instance $m_{\pi, L}=m_{\pi}+c \tilde{g}_{1}\left(m_{\pi} L, T / L\right)$, with $\tilde{g}_{1}(\lambda, \eta)$ a known function [14, 15], $m_{\pi}$ the infinite volume limit, and $c$ a parameter that can be fit to; $L$ is the extent of the lattice in any of the three spatial directions and $T$ is its extent in the temporal direction. The Lattice Higgs Collaboration has taken this approach in order to obtain infinite volume results, where either chiral perturbation theory or hyperscaling relations can be applied. Another answer to this question is given by the theory of finite size scaling, which should reduce to the infinite volume results at sufficiently large $L$. Generally, the form of the scaling function is not known and it is dependent upon the "channel" in which one looks. It has the form

$$
\xi_{L} / L=f\left(m L^{1+\gamma}\right)
$$

where $\xi_{L} \sim 1 / M_{L}, 1 / F_{L}$, correlation lengths in finite volume. One should keep in mind that at finite lattice spacing there will be scaling violations, which are expressed at leading order by an additional term:

$$
\xi_{L} / L=f\left(m L^{y_{m}}\right)+L^{-\omega} g\left(m L^{y_{m}}\right)
$$

Straightforward analysis shows that this is equivalent to the discretization error associated with a nonzero lattice spacing $a \neq 0$. 


\subsection{Schrödinger functional analysis}

In this case one works on a $(L / a)^{4}$ lattice with Dirichlet boundary conditions in the temporal direction, which enforce a background gauge field. For instance for gauge group $S U(2)$,

$$
U_{i}(0, \mathbf{x})=\exp \left[\eta \sigma_{3} a / i L\right], \quad U_{i}(L, \mathbf{x})=\exp \left[(\pi-\eta) \sigma_{3} a / i L\right]
$$

with $\eta=\pi / 4$ and $i=1,2,3$. The fermions are also subject to boundary conditions at $t=0, L$. Boundary counterterms must be introduced in order to get the correct, renormalized continuum limit in the quantum theory [16].

\subsubsection{Running coupling}

The running coupling is obtained from

$$
\bar{g}^{2}=k\left\langle\frac{\partial S}{\partial \eta}\right\rangle^{-1}
$$

The constant $k$ is chosen such that $\bar{g}=g_{0}$, the bare coupling, at leading order in perturbation theory. Evaluating (2.10), one obtains values of $\bar{g}$ nonperturbatively at several values of $g_{0}$ and $L / a$. From this "data" one constructs the step scaling function

$$
\Sigma(u, s, a / L)=\left.\bar{g}^{2}\left(g_{0}, s L / a\right)\right|_{\bar{g}^{2}\left(g_{0}, L / a\right)=u}
$$

where $s>1$ is the scaling parameter, which might be $3 / 2$ or 2 for instance. This relates the running coupling on a lattice of size $(s L / a)^{4}$ to the one on a lattice of size $(L / a)^{4}$, at fixed $g_{0}$, and hence fixed value of $a$.

A few comments are in order here. By taking an increasing sequence of $L / a$ for which $\bar{g}^{2}\left(g_{0}, L / a\right)=u$, with $u, L$ fixed, one obtains

$$
a_{1}>a_{2}>\cdots, \quad g_{0}^{(1)}>g_{0}^{(2)}>\cdots
$$

on the weak coupling side of a putative infrared fixed point, where the continuum limit exists. Thus the continuum limit of the running coupling $\bar{g}^{2}\left(g_{0}, L / a\right)$ is just $\bar{g}^{2}(L)=u$. Since we hold the sequence of bare couplings fixed under the rescaling $L / a \rightarrow s L / a$, the same sequence of lattice spacings occur. Thus the continuum limit obtained from

$$
\sigma(u, s)=\lim _{a / L \rightarrow 0} \Sigma(u, s, a / L)
$$

gives the running coupling $\bar{g}^{2}(s L)$ on a lattice of physical size $s L$ for corresponding value of $\bar{g}^{2}(L)=$ $u$.

Of course we really cannot take $L / a \rightarrow \infty$ so one must fit the sequence of values to an assumed form of the discretization errors, which should be a power series in $a / L$. Thus, having an $\mathscr{O}(a)$ improved action is advantageous in obtaining the continuum limit. It should also be emphasized that a reliable extrapolation, with physics being in the basin of attraction of the ultraviolet fixed 


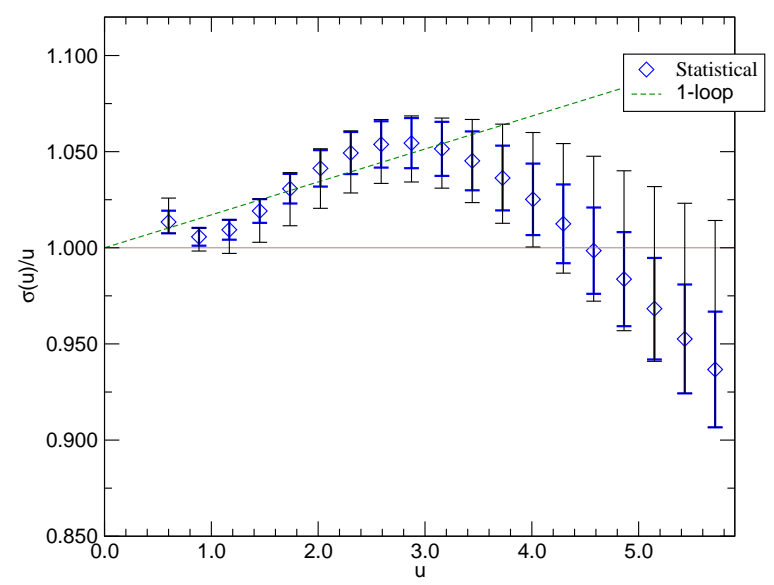

Figure 1: Step scaling function with constant continuum extrapolation, suggesting an infrared fixed point where $\sigma=u$. Taken from [17].

point $g_{0}=0$ where the continuum limit exists, requires that small values of $g_{0}$ actually be part of the calculation. Near the bottom of the conformal window, where the interesting phenomena occur, one is especially concerned about understanding the flow of $\bar{g}$ at large values of $\bar{g}$. This creates a tension in the method. One needs small $g_{0}$ in order to obtain continuum physics (or more importantly a power series in $a$ where only the first few terms are important), and yet one demands a large value of $\bar{g}(L)$. This requires a large value of $L / a$, even more so if the coupling is running slowly. Sometimes it is claimed that step scaling allows one to avoid having to fit a large hierarchy of scales on the same lattice. But if one wants good control over discretization errors and a large value of $\bar{g}(L)$, it is actually necessary to have a large hierarchy of scales on a single lattice. The reliability of the whole method is systematically improved by increasing the size of volumes $(L / a)^{4}$ that are included in the analysis. More work needs to be done in this direction in order to confirm the results of studies that have been done to date, which involve fairly small values of $L / a$. Of course this will require considerably more computing resources than have been employed in the existing studies.

A fixed point is obtained if $\sigma(u, s)=u$ : scaling the lattice by $s$ leaves the running coupling unchanged, $\bar{g}(s L)=\bar{g}(L)$. Thus one typically plots $\sigma(u, s) / u$ versus $u$, and looks for the curve to pass through unity. An example, taken from [17], is shown in Fig. 1.

\subsubsection{Anomalous mass dimension}

The anomalous mass dimension is obtained from the pseudoscalar renormalization constant $Z_{P}$. Because $\bar{\psi} \psi$ and $\bar{\psi} \gamma_{5} t^{a} \psi$ are related by a chiral rotation $\left(t^{a}\right.$ is a generator of the flavor symmetry group), the divergent parts of the $Z$ factors of these two operators are equal. This is enough to make the anomalous dimensions equal. ${ }^{1}$ This is certainly advantageous, since computing the $Z$ factor for the flavor singlet mass operator $\bar{\psi} \psi$ would involve quark-disconnected diagrams, something

\footnotetext{
${ }^{1}$ We thank the authors of [18] for explaining this to us.
} 
that we would like to avoid because of the high computational cost. In the Schrödinger functional approach, $Z_{P}$ is extracted from

$$
Z_{P}\left(g_{0}, L / a\right)=\frac{c \sqrt{C_{\text {boundary }}}}{C_{P}(L / 2)}
$$

where $C_{\text {boundary }}$ is a boundary-to-boundary correlator and $C_{P}\left(t_{0}\right)$ is the pseudoscalar correlation function from the boundary $t=0$ to an interior point $t_{0}$. The constant $c$ is immaterial in what follows. One constructs the step scaling function

$$
\Sigma_{P}(u, s, a / L)=\left.\frac{Z_{P}\left(g_{0}, s L / a\right)}{Z_{P}\left(g_{0}, L / a\right)}\right|_{\bar{g}^{2}\left(g_{0}, L / a\right)=u}
$$

Subsequently a continuum limit is taken

$$
\sigma_{P}(u, s)=\lim _{a / L \rightarrow 0} \Sigma_{P}(u, s, a / L)
$$

One then uses the scaling behavior

$$
\sigma_{P}(u, s)=s^{-\gamma(u)}
$$

to extract the exponent in the vicinity of the fixed point $u_{*}$, provided one exists.

\section{Results for various theories}

In the last five years several theories have been studied using the methods just described. In this section we will summarize the most recent work. We start with a controversial case, which is also the one that has been studied most extensively.

\section{1 $S U(3)$ with twelve fundamental flavors}

At first glance, the claims of the different lattice groups seem quite incompatible with each other. However, under closer examination, what one finds is that different methods, lattice actions and assumptions lead to different conclusions. Twelve flavors becomes a prime example of the difficulty of reaching incontrovertible conclusions in the search for nearly conformal gauge theories on the lattice. Part of the difficulty comes from the fact that this theory is quite close to the edge of the conformal window, so that it is easy to be confused by preliminary results. It also seems that artifacts and deviations from leading order chiral perturbation theory are enhanced by the large number of flavors, which leads to further complications in interpreting results.

One thing that has been learned is that the phase diagram of the twelve flavor theory contains regions with problematic lattice artifacts. For instance, Ref. [19] finds a novel phase where the single site shift symmetry of staggered fermions is spontaneously broken. Finite and zero temperature studies were performed, with the result that two well-separated bulk transitions were identified. The fact that two bulk transitions can occur in the staggered formulation was noticed earlier by the 
authors of $[20,21]$. In this work it was argued that chiral symmetry is restored in the continuum limit, on the weak side of the second bulk transition. The existence of the "second jump" was correlated with Naik improvement of the fermion action in a recent work [22].

In [23] DeGrand has analyzed the Lattice Higgs Collaboration results by a finite-size scaling approach that does not assume a form for the scaling function. He finds an exponent $\gamma \sim 0.35$ across several channels (although with quite large errors) and reasonable fits to the finite-size scaling hypothesis. This can be interpreted as supportive of a conformal or nearly conformal theory in the infrared.

Refs. [24, 25] draw the conclusion that twelve flavors exhibits spontaneous chiral symmetry breaking. They have examined fits to this hypothesis in the Nambu-Goldstone boson (NGB) spectrum, the "pion" decay constant $f_{\pi}$, the chiral condensate, non-NGB hadronic states and a confining static potential (nonzero string tension). Note that in a conformal scenario even the masses of the non-NGB states would go to zero in the chiral limit. They also attempt a fit to the conformal hypothesis with a well-motivated scaling function, finding a low level of confidence in that case: $\chi^{2} /$ d.o.f. $=8.79$. More recently, in [26] they reach similar conclusions, but now with an analysis that does not make any assumptions about the scaling function. The spline based method used here has much smaller errors than [23]. It finds that $\gamma$ extracted from $f_{\pi}$ is significantly different from the other channels.

Ref. [27] performs a fit of the Lattice Higgs Collaboration data with a conformal hypothesis plus additional terms that are motivated by the Schwinger-Dyson equations and additional mass deformations that go beyond the scaling hypothesis of [10-13]. These assumptions are

$$
\begin{aligned}
& M_{X}=C_{X} m^{1 /(1+\gamma)}+D_{X} m, \quad F=C_{F} m^{1 /(1+\gamma)}+D_{F} m, \\
& \langle\bar{\psi} \psi\rangle=A_{C} m+B_{C} m^{(3-\gamma) /(1+\gamma)}+C_{C} m^{3 /(1+\gamma)}+D_{C} m^{3}
\end{aligned}
$$

Neglecting the $D$ terms in these equations, they obtain $\chi^{2} /$ d.o.f. $=2.5$, with $\gamma \approx 0.39$. They find a fairly consistent conformal fit for $\gamma$, though there is some tension in the $f_{\pi}$ channel, as can be seen in Fig. 2. Including the $D$ terms as well as finite volume corrections gives a much better fit, $\chi^{2} /$ d.o.f. $=0.95$.

Ref. [28] finds evidence of an infrared fixed point from the bare step scaling function in Monte Carlo renormalization group studies. For this result an adjoint gauge action term was added and finite volume corrections were more carefully addressed.

At strong coupling, Ref. [29] finds a bulk first order phase transition using unimproved staggered fermions. This phase transition ends at finite bare mass, and they have shown evidence for a second order critical endpoint, where there is a massless scalar hadron. They interpret this as a trivial theory, though further exploration is needed in order to demonstrate this conjecture. On the weak coupling side of the transition, they interpret their results as being supportive of chiral symmetry breaking. However, the study is quite preliminary and requires larger volumes and smaller fermion masses. It is clear that there are artifacts that still need to be addressed, since for instance their linear fit of $m_{\pi}^{2}$ versus $m$ does not pass through $m_{\pi}^{2}=0$ at $m=0$. They have not attempted a 


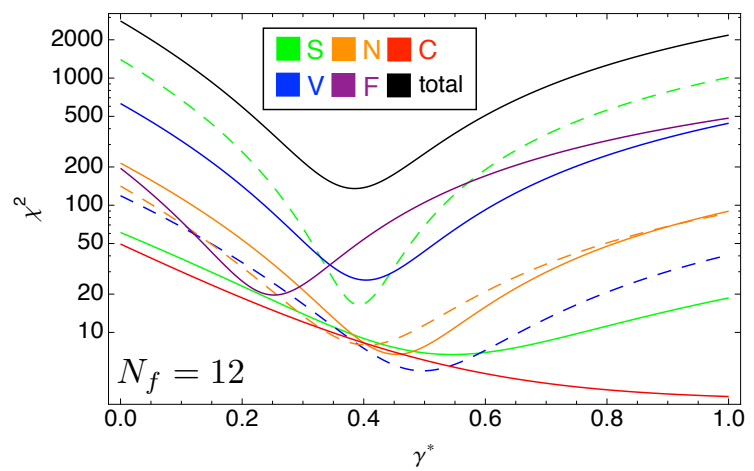

Figure 2: Appelquist et al. [27] fits in various channels.

conformal fit to their data so it is difficult to judge which hypothesis better describes the simulation results.

LatKMI find that their twelve flavor simulations with the HISQ action are supportive of an infrared fixed point [30]. They perform a finite size scaling study that is similar to [23], obtaining $\gamma=0.45(5)$; however, they use a different method for estimating errors, and hence have a much smaller uncertainty. Their Fig. 13 does show some tension between the different channels, where all data are included in the analysis. By slicing up the data in various ways and only fitting parts of it, they are able to relieve the tension. Plots of $f_{\pi} / m_{\pi}$ and $m_{\rho} / m_{\pi}$ versus $m_{\pi}$ show a behavior that is consistent with hyperscaling (approximately constant in the chiral limit), rather than spontaneous chiral symmetry breaking (sharp increase in the chiral limit). However, when they attempt a fit to chiral perturbation theory using only data with small masses $m \leq 0.08$, they find $\chi^{2} /$ d.o.f. $=0.88$. They also find a nonzero $f_{\pi}$ in the chiral limit with a good fit to chiral perturbation theory. They caution, however, that $m_{\pi} / f_{\pi}$ is outside the range of validity of chiral perturbation theory.

\section{2 $S U(3)$ with two flavors of sextet fermions}

The sextet is the two-index symmetric representation. It is thus in the same family of theories as the adjoint representation for SU(2) (which has been studied extensively and found to be conformal in the infrared), and the decuplet representation in $\mathrm{SU}(4)$. The sextet is in some sense the most minimal technicolor model, as there are precisely the right number of NGBs to be eaten by the $W^{ \pm}, Z$, leaving no extra NGBs to be given mass by chiral symmetry violating terms.

Ref. [24] finds chiral symmetry breaking, using fits to this hypothesis in the NGB spectrum, the "pion" decay constant $f_{\pi}$ and the chiral condensate. In the follow-up work [26] they find that fitting to the conformal hypothesis gives very different values of $\gamma$ in the various channels. Hence they conclude that the sextet model does not have an infrared fixed point and is chirally broken.

Ref. [31] finds evidence of an infrared fixed point, though they state that their results could also be explained by a walking theory. They use the Schrödinger functional method and measure a discrete beta function, which appears to pass through zero. Interestingly, they also find a value of $\gamma$ 
that is quite similar to the $\mathrm{SU}(2)$ and $\mathrm{SU}(4)$ cases with two flavors in the the two-index symmetric representation.

Ref. $[32,33]$ continues the study of the finite temperature transition. The idea is that if the critical coupling $\beta_{c}$ scales properly with the lattice temporal extent $N_{t}$, then chiral symmetry is spontaneously broken in the continuum theory at zero temperature. More definite values for the peak of the chiral susceptibility are needed at more values of $N_{t}$ in order to reach firm conclusions.

Ref. [34] studies the possibility of a "Higgs impostor" in the sextet model. While they outline the study of flavor singlet $0^{++}$states in this theory, the results in this work are mainly further evidence in support of spontaneous chiral symmetry breaking, and evidence against a mass deformed conformal field theory.

\section{3 $S U(3)$ with ten fundamental flavors}

This theory is believed to be near the bottom of the conformal window. Eight flavors is generally accepted as being below the conformal window. Many studies have concluded that twelve flavors is inside the conformal window. If we accept these conclusions, then ten flavors may be a walking theory. The lattice studies that have been performed to date find a fairly large value of $\gamma$, close to unity, as required for phenomenological applications. Thus this theory is well motivated as a theory for extensive study. Of course, it is more difficult since the number of flavors is not a multiple of four. For this reason non-rooted staggered fermions cannot be used.

Early ten flavor work includes $[35,36]$ which report results of a Schrödinger functional study. They found behavior that they concluded was consistent with an infrared fixed point and large anomalous mass dimension $\gamma$. However, the results are quite preliminary. This study used unimproved Wilson fermions (no clover improvement term, and hence $\mathscr{O}(a)$ discretization errors), and the plaquette gauge action without boundary counterterms. This simple set-up obviously leaves room for future improvements with systematic errors under better control. As usual in the many flavors theories, a bulk transition is encountered at strong coupling, which for them occurs at $\beta=6 / g_{0}^{2}=4.4$. The lattices used are fairly small: $6^{4}, 8^{4}$ and $12^{4}$. It will be interesting to see what more extensive and refined Schrödinger functional studies reveal in the ten flavor theory.

Ref. [37] is recent and finds that fits to the mass deformed conformal hypothesis are favored. Interestingly they find $\gamma \approx 1$ for the exponent, the value preferred by solutions to flavor-changing neutral current problems. They motivate their study of $N_{f}=10$ based on their positive findings in the $N_{f}=6$ case: an enhanced condensate [38] and a reduced S-parameter [39]. The good conformal fits that they are able to achieve could also correspond to a nearly conformal theory. It will be very interesting to see their analysis of the S-parameter in the ten flavor theory, once it is available. Based on their $N_{f}=6$ results, it may be quite small due to degeneracy between the vector and pseudovector mesons. The $N_{f}=10$ results are from a single volume $\left(32^{3} \times 64\right)$, and in order to probe the small mass regime, larger volumes will be required. This is an expensive proposition, as the authors of this work have elected to use domain wall fermions due to their superior chiral properties. While this allows them to measure the chiral condensate, and to study $N_{f}=10$ without 


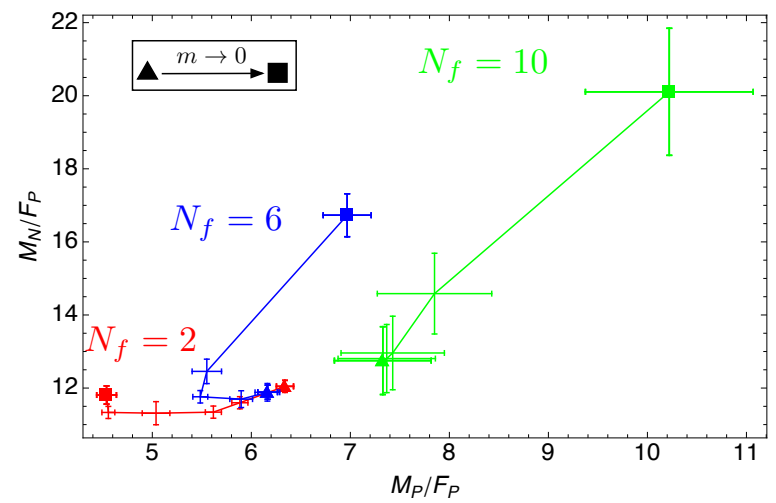

Figure 3: Edinburgh-style plot to detect mass at which finite volume corrections set in. This occurs when the data begins to move up and to the right. For ten flavors, it can be seen that the three largest masses are largely free of finite volume corrections. Figure from [37].

"rooting," the computational cost is daunting. Due to the problem of fixed topology, the authors had to study observables in both ordered start configurations (zero topological charge sector) and disordered start configurations (large topological charge sectors). The difference between the two results was included as a (significant, 3\%-7\%) systematic error. They are working to measure topological susceptibility and use this to calculate corrections that come from working in fixed topological sectors. This is an illustration of a problem that occurs when chiral lattice fermions are used with a large number of flavors: the fermion determinant raised to the $N_{f}$ power is prohibitively small near a topological transition.

In spite of working on a fixed lattice volume $32^{3} \times 64$, they are able to quantify finite volume effects using "Edinburgh-style plots." Here one plots ratios of physical observables versus each other. On the vertical axis is plotted $m_{N} / f_{\pi}$ while on the horizontal axis is plotted $m_{\pi} / f_{\pi}$. As $m \rightarrow 0$, when in the infinite volume regime, the points move to the left. But once finite volume corrections start to take over, the points move up and to the right. This can then be used as an indicator of the range of masses for which finite volume corrections are under control. Examples of this behavior are shown in Fig. 3.

\section{4 $S U(2)$ with fundamental flavors}

Works on this topic appearing in these proceedings include [40,41]. Older work is [17, 42] which studied $N_{f}=6$ using the Schrödinger functional method, finding a slowly running coupling and $0.135<\gamma<1.03$. In that case $L / a=6,8,10,12,14,16$ was simulated. However, $L / a=6$ was discarded because large lattice artifacts were found.

A more recent work is [43], which studies $N_{f}=4,6$ and 10. They also use the Schrödinger functional, and find for six flavors that the results are inconclusive as to whether or not there is an infrared fixed point. On the other hand for four flavors the theory is QCD-like and in the ten flavors case the theory has a Banks-Zaks fixed point behavior. 


\begin{tabular}{|l|l|l|l|l|}
\hline$\beta_{L}$ & $L / a=6$ & $L / a=8$ & $L / a=12$ & $L / a=16$ \\
\hline 8 & $0.4700(2)$ & $0.4706(4)$ & $0.4705(5)$ & $0.4707(10)$ \\
6 & $0.6148(3)$ & $0.6159(5)$ & $0.6180(9)$ & $0.6181(19)$ \\
4 & $0.8897(9)$ & $0.8897(13)$ & $0.895(4)$ & $0.895(3)$ \\
3 & $1.1528(16)$ & $1.156(3)$ & $1.150(2)$ & $1.146(4)$ \\
2 & $1.651(4)$ & $1.653(5)$ & $1.637(6)$ & $1.624(13)$ \\
1.7 & $1.924(4)$ & $1.907(5)$ & $1.905(11)$ & $1.896(13)$ \\
1.5 & $2.183(3)$ & $2.137(7)$ & $2.116(11)$ & $2.10(2)$ \\
1.3 & $2.542(8)$ & $2.473(9)$ & $2.382(11)$ & $2.37(2)$ \\
1 & $4.03(2)$ & $3.55(2)$ & $3.23(3)$ & $3.09(4)$ \\
\hline
\end{tabular}

Table 1: The measured values [43] of $\bar{g}^{2}$ at each $\beta_{L}=4 / g_{0}^{2}$ and $L / a$ with ten flavors of fundamental representations fermions in the gauge group $\mathrm{SU}(2)$.

Actually, an interesting phenomenon occurs for ten flavors in [43], observed by carefully examining Table 4 of their work, which is reproduced here as Table 1. It can be seen that the running coupling does not change with increasing $L / a$ for $\beta_{L}=4 / g_{0}^{2}=3,4,6$ and 8 . However, this is not fixed point behavior, since $\bar{g}$ takes on markedly different values over this wide range of bare $g_{0}$. The correct understanding of this is in terms of a very small one-loop contribution to the $\beta$ function and a negligible higher order contribution. At one loop

$$
\frac{1}{g^{2}(6)}-\frac{1}{g^{2}(16)}=\frac{1}{48 \pi^{2}} \ln \frac{8}{3} \Rightarrow \frac{g^{2}(16)-g^{2}(6)}{g^{2}(6)} \approx g^{2}(6) \times 2.07 \times 10^{-3}
$$

so that the relative change in $\bar{g}^{2}$ is far less than a percent in going from $L / a=6$ to $L / a=16$. This illustrates that it is possible to fool oneself into believing that fixed point behavior is occuring, when it is actually just a very small one-loop coefficient and relative weak coupling that is causing the phenomenon. This will always happen near the top of the conformal window, since the one-loop coefficient is about to change sign, and is hence close to zero.

\section{5 $S U(2)$ with two adjoint flavors}

This theory is often referred to as "minimal walking technicolor (MWTC)" in the literature. It has been extensively studied and here we only mention recent work. A recent Schrödinger functional study finds a zero of the discrete $\beta$ function [44]. The Monte Carlo renormalization group method finds a small anomalous mass dimension $\gamma<0.6$, but is unable to reach firm conclusions on the bare step scaling function [45-47]. Finite size scaling from spectral data finds $\gamma=0.50(26)$, compatible with other studies, and again too small for phenomenological purposes [48]. Analysis of the Dirac eigenmode spectrum leads to similar conclusions for $\gamma$ and the most accurate value to date, $\gamma=0.371(20)$, Ref. [49]. In [50,51] we found that using the Creutz ratio definition of the running coupling, the theory either had no running or backward running in the vicinity of bare coupling $\beta=2.25$. Other recent work includes nonperturbative improvement [52]. All results on $\gamma$ are summarized in Table 2. In all cases it is too small for phenomenological purposes. 


\begin{tabular}{|c|c|}
\hline \hline Method & $\gamma$ \\
\hline \hline SF [53] & $0.05<\gamma<0.56$ \\
\hline SF [44] & $0.31 \pm 0.06$ \\
\hline Perturbative 4-loop [54,55] & 0.500 \\
\hline Schwinger-Dyson [55] & 0.653 \\
\hline All-orders hypothesis [56] & 0.46 \\
\hline MCRG [47] & $-0.6<\gamma<0.6$ \\
\hline FSS [57] & $0.05<\gamma<0.20$ \\
\hline FSS [58] & $0.22 \pm 0.06$ \\
\hline FSS [48] & $0.50 \pm 0.26$ \\
\hline Dirac eigenvalues [49] & $0.371 \pm 0.020$ \\
\hline \hline
\end{tabular}

Table 2: Summary of all MWTC results for the anomalous mass dimension. SF is Schrödinger functional, MCRG is Monte Carlo renormalization group and FSS is finite size scaling.

\section{6 $S U(4)$ with two flavors of decuplet fermions}

This theory was studied using Schrödinger functional methods in [18]. Similar to SU(2) and SU(3) with two-index symmetric representation fermions, a zero in the discrete beta function is found. The conclusion reached by the authors is that the theory flows to an infrared fixed point.

\subsection{Two-dimensional $O(3) \sigma$ model as a walking theory}

With a $\theta$ term, the $O(3) \sigma$ model can be continuously deformed away from a conformal theory in the infrared. Thus it is like a theory with $N_{f}$ flavors where $N_{f}$ is a continuous parameter. This analogy was first suggested in [59], and subsequently studied in detail in [60]. The approximate zero of the $\beta$ function at a nonzero coupling can be seen in Fig. 4 .

\section{Conclusion}

Subsequent experimental work at the Large Hadron Collider will determine the $J^{P C}$ quantum numbers of the new boson that has been discovered. Greater certainty regarding rates in various decay channels will be obtained as integrated luminosity is accumulated and analysis techniques refined. A detailed understanding of these properties will give us greater guidance in answering the question of whether or not this boson originates from a theory with compositeness at a higher scale. This in turn will tell us whether or not conformal theories have anything to do with $\mathrm{TeV}$ scale physics. In the meantime, speculations will continue about this possibility and lattice studies of models inspired by this scenario will be on-going. We are gradually learning more about conformal and nearly conformal theories arising from new strong interactions, but it has proven to be a challenging endeavor because of the many subtleties involved. Whether or not nature "chooses" to make use of conformal or nearly conformal theories at energies greater than the electroweak 


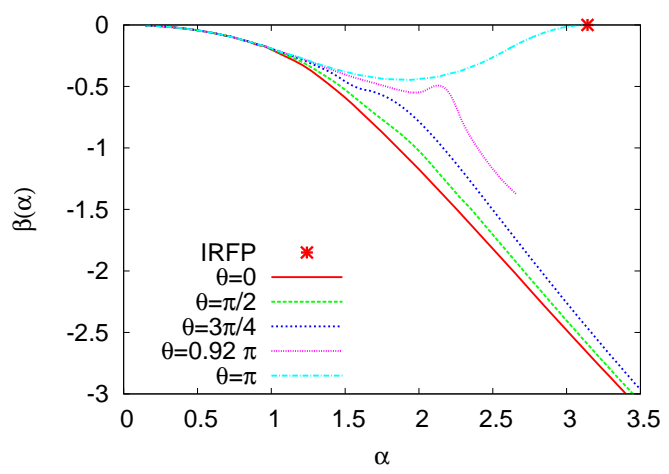

Figure 4: $\beta$ function in the two-dimensional $O(3)$ model with various values of $\theta$. It can be seen that for $\theta$ near the critical value, the walking behavior sets in, where there is an approximate zero of the $\beta$ function at a nonzero coupling.

scale, they are nevertheless quite interesting from a theoretical perspective. To a certain degree, lattice gauge theory has proven its worth in that it has given in some cases strong indications about which theories lie within the conformal window and what is the range of values that occurs for the exponent $\gamma$. Given the short time period over which these studies have been conducted, and the modest resources that have been devoted to them, it is a triumph of this first principles approach to strongly coupled quantum field theory that it has been able to make a number of compelling statements. However, it is clear that more work is needed in order to reconcile the various studies near the bottom of the conformal window. This is most obvious in the case of $S U$ (3) lattice gauge theory with twelve fundamental flavors of fermions. A better understanding of the strengths and weaknesses of various methods is needed, so that a consensus can be reached about how much weight to give the different results. Many of the groups have only performed preliminary studies on relatively small volumes, and so it is important for them to refine and improve their work before we attempt to draw final conclusions. It will hopefully be the case that we will also see innovations over the next few years that will lead to more definitive answers in the theories that we are studying. It is hoped that more results with phenomenological implications will be obtained as time goes on.

\section{References}

[1] L. Susskind, The Anthropic landscape of string theory, hep-th/0302219.

[2] B. S. Acharya, G. Kane, and P. Kumar, Compactified String Theories - Generic Predictions for Particle Physics, Int.J.Mod.Phys. A27 (2012) 1230012, [arXiv:1204.2795].

[3] T. Gherghetta and J. Giedt, Bulk fields in AdS(5) from probe D7 branes, Phys.Rev. D74 (2006) 066007, [hep-th/0605212].

[4] T. Appelquist and J. Terning, An Extended technicolor model, Phys.Rev. D50 (1994) 2116-2126, [hep-ph/9311320]. 
[5] ATLAS Collaboration Collaboration, G. Aad et al., Observation of a new particle in the search for the Standard Model Higgs boson with the ATLAS detector at the LHC, Phys.Lett. B716 (2012) 1-29, [arXiv:1207.7214].

[6] CMS Collaboration Collaboration, S. Chatrchyan et al., Observation of a new boson at a mass of 125 GeV with the CMS experiment at the LHC, Phys.Lett. B716 (2012) 30-61, [arXiv: 1207.7235 ].

[7] B. Bellazzini, C. Csaki, J. Hubisz, J. Serra, and J. Terning, A Higgslike Dilaton, arXiv:1209.3299.

[8] W. E. Caswell, Asymptotic Behavior of Nonabelian Gauge Theories to Two Loop Order, Phys.Rev.Lett. 33 (1974) 244.

[9] T. Banks and A. Zaks, On the Phase Structure of Vector-Like Gauge Theories with Massless Fermions, Nucl.Phys. B196 (1982) 189.

[10] V. Miransky, Dynamics in the conformal window in QCD like theories, Phys.Rev. D59 (1999) 105003, [hep-ph/9812350].

[11] M. A. Luty, Strong Conformal Dynamics at the LHC and on the Lattice, JHEP 0904 (2009) 050, [arXiv:0806.1235].

[12] L. Del Debbio and R. Zwicky, Hyperscaling relations in mass-deformed conformal gauge theories, Phys.Rev. D82 (2010) 014502, [arXiv: 1005 .2371].

[13] L. Del Debbio and R. Zwicky, Scaling relations for the entire spectrum in mass-deformed conformal gauge theories, Phys.Lett. B700 (2011) 217-220, [arXiv:1009.2894].

[14] H. Leutwyler, Energy levels of light quarks confined to a box, Phys.Lett. B189 (1987) 197.

[15] M. Luscher, Volume Dependence of the Energy Spectrum in Massive Quantum Field Theories. 1. Stable Particle States, Commun.Math.Phys. 104 (1986) 177.

[16] S. Sint, One loop renormalization of the QCD Schrodinger functional, Nucl.Phys. B451 (1995) 416-444, [hep-lat/9504005].

[17] F. Bursa, L. Del Debbio, L. Keegan, C. Pica, and T. Pickup, Mass anomalous dimension and running of the coupling in SU(2) with six fundamental fermions, PoS LATTICE2010 (2010) 070, [arXiv:1010.0901].

[18] T. DeGrand, Y. Shamir, and B. Svetitsky, SU(4) lattice gauge theory with decuplet fermions: Schrodinger functional analysis, Phys.Rev. D85 (2012) 074506, [arXiv: 1202 . 2675].

[19] A. Cheng, A. Hasenfratz, and D. Schaich, Novel phase in SU(3) lattice gauge theory with 12 light fermions, Phys.Rev. D85 (2012) 094509, [arXiv:1111.2317].

[20] A. Deuzeman, E. Pallante, and M. P. Lombardo, The Bulk transition of many-flavour QCD and the search for a UVFP at strong coupling, PoS LATTICE2010 (2010) 067, [arXiv: 1012.5971 ].

[21] A. Deuzeman, M. P. Lombardo, T. N. da Silva, and E. Pallante, Bulk transitions of twelve flavor QCD and $U_{A}(1)$ symmetry, PoS LATTICE2011 (2011) 321, [arXiv:1111. 2590].

[22] A. Deuzeman, M. P. Lombardo, T. N. da Silva, and E. Pallante, The bulk transition of QCD with twelve flavors and the role of improvement, arXiv:1209.5720. 
[23] T. DeGrand, Finite-size scaling tests for spectra in SU(3) lattice gauge theory coupled to 12 fundamental flavor fermions, Phys.Rev. D84 (2011) 116901, [arXiv:1109.1237].

[24] Z. Fodor, K. Holland, J. Kuti, D. Nogradi, and C. Schroeder, Chiral symmetry breaking in fundamental and sextet fermion representations of SU(3) color, arXiv: 1103.5998.

[25] Z. Fodor, K. Holland, J. Kuti, D. Nogradi, C. Schroeder, et al., Twelve massless flavors and three colors below the conformal window, Phys.Lett. B703 (2011) 348-358, [arXiv: 1104 . 3124 ].

[26] Z. Fodor, K. Holland, J. Kuti, D. Nogradi, C. Schroeder, et al., Twelve fundamental and two sextet fermion flavors, arXiv:1205.1878.

[27] T. Appelquist, G. Fleming, M. Lin, E. Neil, and D. Schaich, Lattice Simulations and Infrared Conformality, Phys.Rev. D84 (2011) 054501, [arXiv:1106.2148].

[28] A. Hasenfratz, Infrared fixed point of the 12-fermion SU(3) gauge model based on 2-lattice MCRG matching, Phys.Rev.Lett. 108 (2012) 061601, [arXiv:1106.5293].

[29] X.-Y. Jin and R. D. Mawhinney, Lattice QCD with 12 Degenerate Quark Flavors, PoS LATTICE2011 (2011) 066, [arXiv:1203.5855].

[30] Y. Aoki, T. Aoyama, M. Kurachi, T. Maskawa, K.-i. Nagai, et al., Lattice study of conformality in twelve-flavor QCD, Phys.Rev. D86 (2012) 054506, [arXiv: 1207.3060 ].

[31] T. DeGrand, Y. Shamir, and B. Svetitsky, Mass anomalous dimension in sextet QCD, arXiv:1201.0935.

[32] D. Sinclair and J. Kogut, The chiral phase transition for QCD with sextet quarks, PoS LATTICE2011 (2011) 090, [arXiv:1111.2319].

[33] J. Kogut and D. Sinclair, Thermodynamics of lattice QCD with 3 flavours of colour-sextet quarks, Phys.Rev. D85 (2012) 054505, [arXiv:1111.3353].

[34] Z. Fodor, K. Holland, J. Kuti, D. Nogradi, C. Schroeder, et al., Can the nearly conformal sextet gauge model hide the Higgs impostor?, arXiv:1209.0391.

[35] N. Yamada, M. Hayakawa, K.-I. Ishikawa, Y. Osaki, S. Takeda, et al., Study of the running coupling constant in 10-flavor QCD with the Schródinger functional method, arXiv: 1003.3288.

[36] M. Hayakawa, K.-I. Ishikawa, Y. Osaki, S. Takeda, S. Uno, et al., Running coupling constant of ten-flavor QCD with the Schródinger functional method, Phys.Rev. D83 (2011) 074509, [arXiv:1011.2577].

[37] T. Appelquist, R. C. Brower, M. I. Buchoff, M. Cheng, S. D. Cohen, et al., Approaching Conformality with Ten Flavors, arXiv:1204.6000.

[38] LSD Collaboration Collaboration, T. Appelquist et al., Toward TeV Conformality, Phys.Rev.Lett. 104 (2010) 071601, [arXiv:0910.2224].

[39] LSD Collaboration Collaboration, T. Appelquist et al., Parity Doubling and the S Parameter Below the Conformal Window, Phys.Rev.Lett. 106 (2011) 231601, [arXiv: 1009.5967 ].

[40] T. Karavirta, K. tuominen, J. Rantaharju, and K. Rummukainen, Mapping the Conformal Window: SU(2) with 4, 6 and 10 flavors of fermions, arXiv:1210.4228. 
[41] M. Hayakawa, K.-I. Ishikawa, Y. Osaki, S. Takeda, and N. Yamada, Lattice study on two-color QCD with six flavors of dynamical quarks, arXiv:1210.4985.

[42] F. Bursa, L. Del Debbio, L. Keegan, C. Pica, and T. Pickup, Mass anomalous dimension in SU(2) with six fundamental fermions, Phys.Lett. B696 (2011) 374-379, [arXiv: 1007 . 3067$].$

[43] T. Karavirta, J. Rantaharju, K. Rummukainen, and K. Tuominen, Determining the conformal window: $S U(2)$ gauge theory with $N_{f}=4,6$ and 10 fermion flavours, JHEP 1205 (2012) 003, [arXiv:1111.4104].

[44] T. DeGrand, Y. Shamir, and B. Svetitsky, Infrared fixed point in SU(2) gauge theory with adjoint fermions, Phys.Rev. D83 (2011) 074507, [arXiv: 1102 . 2843].

[45] S. Catterall, L. Del Debbio, J. Giedt, and L. Keegan, MCRG Minimal Walking Technicolor, PoS LATTICE2010 (2010) 057, [arXiv: 1010 . 5909].

[46] S. Catterall, L. Del Debbio, J. Giedt, and L. Keegan, Systematic Errors of the MCRG Method, PoS LATTICE2011 (2011) 068, [arXiv:1110.1660].

[47] S. Catterall, L. Del Debbio, J. Giedt, and L. Keegan, MCRG Minimal Walking Technicolor, Phys.Rev. D85 (2012) 094501, [arXiv: 1108.3794$]$.

[48] J. Giedt and E. Weinberg, Finite size scaling in minimal walking technicolor, Phys.Rev. D85 (2012) 097503, [arXiv:1201.6262].

[49] A. Patella, A precise determination of the $\bar{\psi} \psi$ anomalous dimension in conformal gauge theories, Phys.Rev. D86 (2012) 025006, [arXiv:1204.4432].

[50] J. Giedt and E. Weinberg, Backward running or absence of running from Creutz ratios, Phys.Rev. D84 (2011) 074501, [arXiv:1105.0607].

[51] J. Giedt and E. Weinberg, Backward running or absence of running from Creutz ratios, PoS LATTICE2011 (2011) 238.

[52] T. Karavirta, A. Mykkanen, J. Rantaharju, K. Rummukainen, and K. Tuominen, Nonperturbative improvement of $S U(2)$ lattice gauge theory with adjoint or fundamental flavors, JHEP 1106 (2011) 061, [arXiv:1101.0154].

[53] F. Bursa, L. Del Debbio, L. Keegan, C. Pica, and T. Pickup, Mass anomalous dimension in SU(2) with two adjoint fermions, Phys.Rev. D81 (2010) 014505, [arXiv: 0910 . 4535].

[54] C. Pica and F. Sannino, UV and IR Zeros of Gauge Theories at The Four Loop Order and Beyond, Phys.Rev. D83 (2011) 035013, [arXiv:1011.5917].

[55] T. A. Ryttov and R. Shrock, Higher-Loop Corrections to the Infrared Evolution of a Gauge Theory with Fermions, Phys.Rev. D83 (2011) 056011, [arXiv: 1011.4542 ].

[56] C. Pica and F. Sannino, Beta Function and Anomalous Dimensions, Phys.Rev. D83 (2011) 116001, [arXiv:1011.3832].

[57] L. Del Debbio, B. Lucini, A. Patella, C. Pica, and A. Rago, Mesonic spectroscopy of Minimal Walking Technicolor, Phys.Rev. D82 (2010) 014509, [arXiv: 1004 . 3197].

[58] L. Del Debbio, B. Lucini, A. Patella, C. Pica, and A. Rago, The infrared dynamics of Minimal Walking Technicolor, Phys.Rev. D82 (2010) 014510, [arXiv: 1004.320 6]. 
[59] D. Nogradi, An ideal toy model for confining, walking and conformal gauge theories: the O(3) sigma model with theta-term, JHEP 1205 (2012) 089, [arXiv: 1202.4616].

[60] P. de Forcrand, M. Pepe, and U.-J. Wiese, Walking near a Conformal Fixed Point: the 2-d O(3) Model at theta near pi as a Test Case, arXiv:1204.4913. 